\title{
MOSCAS-DAS-FRUTAS (DIPTERA: TEPHRITIDAE) EM UM POMAR DE GOIABEIRA, NO SEMIÁRIDO BRASILEIRO ${ }^{1}$
}

\author{
ELTON LUCIO ARAUJO2, JULIANA DA COSTA RIBEIRO 3 , \\ MARCONE CESAR MENDONÇA CHAGAS ${ }^{4}$, VIVIAN SIQUEIRA DUTRA ${ }^{5}$, \\ JANISETE GOMES SILVA ${ }^{6}$
}

RESUMO - As moscas-das-frutas (Diptera: Tephritidae) são pragas-chave na cultura da goiabeira Psidium guajava L., com predominância de diferentes espécies de acordo com a região produtora no Brasil. Os objetivos do presente trabalho foram conhecer a diversidade e analisar parâmetros faunísticos das moscasdas-frutas obtidas em um pomar de goiabeira, no município de Cruzeta, Rio Grande do Norte, situado no semiárido brasileiro. As moscas-das-frutas foram coletadas semanalmente, com auxílio de armadilhas McPhail, tendo como atrativo proteína hidrolisada a $5 \% \mathrm{v} / \mathrm{v}$. Foram registradas cinco espécies no pomar estudado: Ceratitis capitata (Wied.), Anastrepha zenildae Zucchi, Anastrepha sororcula Zucchi, Anastrepha obliqua (Macquart) e Anastrepha dissimilis Stone. Ceratitis capitata foi a espécie mais frequente, constante e dominante, considerada como uma praga invasiva, potencial em pomares de goiabeira no semiárido brasileiro. Termos para indexação: Psidium guajava, moscas-das-frutas, semiárido, análise faunistica.

\section{FRUIT FLIES (DIPTERA: TEPHRITIDAE) ON A GUAVA ORCHARD, IN THE BRAZILIAN SEMIARID}

\begin{abstract}
Fruit flies (Diptera: Tephritidae) are key pests of guava Psidium guajava L. crops, with different dominant species according to the Brazilian growth region The objectives of this study were to determine the fruit fly diversity and submit the results of flies captured in guava orchard from the municipality of Cruzeta, state of Rio Grande do Norte, Brazilian semiarid to the faunistic analysis. Fruit flies were collected weekly with McPhail traps using protein hydrolisate at $5 \% \mathrm{v} / \mathrm{v}$. Five species were recorded in the orchard: Ceratitis capitata (Wied.), Anastrepha zenildae Zucchi, Anastrepha sororcula Zucchi, Anastrepha obliqua (Macquart) and Anastrepha dissimilis Stone. Ceratitis capitata was the most frequent, constant, and dominant species and could be considered an invasive species with potential to become pest on guava orchards in the Brazilian semiarid.
\end{abstract}

Index terms: Psidium guajava, fruit flies, semiarid, faunistic analyses.

\section{INTRODUÇÃO}

A goiabeira (Psidium guajava L.) (Myrtaceae), originária dos trópicos americanos, é uma das frutíferas mais comuns e economicamente importantes nas regiões tropicais e subtropicais do mundo (SINGH; PAL, 2008). No Brasil, a goiabeira é cultivada em escala comercial em praticamente todas as regiões (PEREIRA; RYOSUKE, 2011). Na região Nordeste, onde predomina o clima semiárido, o plantio dessa cultura vem sendo ampliado, principalmente devido às condições climáticas favoráveis e às avançadas técnicas de irrigação.

No entanto, a ocorrência de pragas, principalmente as moscas-das-frutas (Diptera: Tephritidae), é um fator limitante para produção de goiaba. As larvas desses tefritídeos consomem a polpa do fruto, tornando-os impróprios para o consumo in natura e até mesmo para a industrialização. Muitas frutíferas da família Myrtaceae são hospedeiras

\footnotetext{
'(Trabalho 231-12). Recebido em: 27-08-2012. Aceito para publicação em: 18-02-2013.

${ }^{2}$ Doutor em Entomologia. Professor da Universidade Federal Rural do Semi-Árido, Km 47 da BR 110, Caixa Postal 137, 59625-900, Mossoró-RN, Brasil. E-mail: elton@ufersa.edu.br

${ }^{3}$ Graduada em Agronomia. Estagiária da Universidade Federal Rural do Semi-Árido, Km 47 da BR 110, Caixa Postal 137, 59625-900, Mossoró-RN, Brasil.E-mail:juh_ribeiro@hotmail.com

${ }^{4}$ Doutor em Entomologia. Pesquisador da EMBRAPA/EMPARN - Empresa de Pesquisa Agropecuária do Rio Grande do Norte, Av. Elisa Branco Pereira dos Santos, Caixa Postal 188, 59158-160, Parnamirim, RN, Brasil. E-mail: marcone@rn.gov.br

${ }^{5}$ Doutora em Entomologia. Instituto Nacional de Pesquisas da Amazônia - INPA, CP 478, 69011-970, Manaus-AM, Brasil. E-mail: dutrasv@gmail.com

${ }^{6}$ Doutora em Ciências Biológicas (Biologia/Genética). Professora da Universidade Estadual de Santa Cruz, Rodovia Ilhéus/Itabuna km 16, 45650-000, Ilhéus-BA, Brasil. E-mail: jgs10@uol.com.br
} 
primárias de moscas-das-frutas (SILVA et al., 2010; SILVA et al., 2011; BIRK; ALUJA, 2011). No Brasil, Ceratitis capitata (Wied.) e mais de 11 espécies de Anastrepha já foram relatadas infestando frutos de goiaba (ZUCCHI, 2007; SOUZA FILHO et al., 2009)

A predominância de espécies de moscas-das-frutas nos pomares de goiabeira é variável entre as regiões do Brasil. Nas regiões Sul e Sudeste, a espécie mais comum em goiaba é Anastrepha fraterculus (Wied.) (RAGA et al., 2006). Na região Centro-Oeste, Anastrepha sororcula Zucchi e C. capitata são frequentes em goiaba (NICÁCIO; UCHÔA, 2011). Na região Norte, Anastrepha striata Schiner é a mais comum (PEREIRA et al., 2010). Nas regiões semiáridas de Minas Gerais (sudeste) e interior do Nordeste, Anastrepha zenildae Zucchi é a espécie predominante (ARAUJO et al., 2005; ALVARENGA et al., 2009). No litoral da região Nordeste, $A$. fraterculus é mais comum (SILVA et al., 2011). De acordo com Silva et al. (2010), diversos fatores, como o clima, altitude, localização geográfica, hospedeiros e pomares adjacentes, podem influenciar na diversidade e dominância de espécies de moscas-das-frutas nos pomares.

Contudo, para estabelecer-se qualquer programa de manejo integrado de moscas-das-frutas nos pomares, torna-se necessário o prévio conhecimento de aspectos ecológicos desses tefritídeos, como, por exemplo, a diversidade de espécies presente nos pomares, frequência, dominância, constância das espécies, entre outros parâmetros faunísticos (AGUIAR-MENEZES et al., 2008).

Portanto, considerando-se a importância da cultura da goiabeira, escassez de estudos no semiárido brasileiro e o fato de este ecossistema possuir características climáticas particulares e flora endêmica, o presente trabalho tem como objetivo principal conhecer a diversidade de moscas-das-frutas associadas à cultura da goiabeira, em um pomar localizado numa região semiárida do Brasil, e avaliar a dominância, constância e frequência das espécies desses tefritídeos.

\section{MATERIAL E MÉTODOS}

Este trabalho foi realizado no período de janeiro/2008 a dezembro/2009 em um pomar comercial de goiabeira cv. Paluma, situado no município de Cruzeta (6²19'38,23" S e 36 $44^{\prime} ' 15,92^{\prime \prime}$ W, com 232 $\mathrm{m}$ de altitude), inserido na microrregião do Seridó, Estado do Rio Grande do Norte, Brasil. A microrregião do Seridó caracteriza-se por apresentar clima semiárido, seco, muito quente e estação chuvosa no verão (Bswh - Köeppen), vegetação classificada como caatinga hiperxerófila, com médias anuais de temperatura, umidade relativa do ar e precipitação pluvial de $30^{\circ} \mathrm{C}, 50 \%$ e $600 \mathrm{~mm}$, respectivamente (AMORIM et al., 2005). A área do pomar estudado corresponde a nove hectares com plantas em torno de cinco anos de idade, sendo estas submetidas a tratamento fitossanitário apenas para o controle de psilídeo Triozoida sp. e besouro-amarelo Costalimaita ferruginea vulgata Lefevre.

Para a captura das moscas-das-frutas, foram utilizadas armadilhas do tipo McPhail de base amarela, com solução de proteína hidrolisada de milho a $5 \% \mathrm{v} / \mathrm{v}$ como atrativo alimentar. As armadilhas foram distribuídas na proporção de uma (1) armadilha por hectare, totalizando nove armadilhas, instaladas a uma altura de 1,5 metro do solo, no interior da copa da planta, equidistantes 100 metros uma das outras.

Semanalmente, cada armadilha foi abastecida com $500 \mathrm{~mL}$ da solução atrativa, ocasião na qual os insetos capturados foram coletados e depositados em recipientes plásticos, contendo álcool a $70 \%$, e transportados para o Laboratório de Entomologia Aplicada da UFERSA, Mossoró (RN). No laboratório, os insetos foram triados, e as moscas-das-frutas obtidas foram sexadas, contadas e identificadas.

Durante a condução do trabalho, também foram realizadas coletas aleatórias de frutos de goiaba maduros para verificar quais espécies de moscas-das-frutas utilizam este fruto como hospedeiro, na região. Os frutos coletados foram acondicionados em bandejas plásticas, contendo uma camada de três centimetros de areia lavada. Após 10 dias, a areia foi peneirada para a obtenção dos pupários. Os pupários obtidos foram acondicionados em recipientes plásticos, cobertos por voile, onde permaneceram até a emergência das moscas-das-frutas. Os adultos obtidos foram acondicionados em álcool a 70\% e posteriormente identificados. Os exemplares foram identificados, analisando-se o padrão de coloração do corpo, nervuras das asas e as características morfométricas do ápice do acúleo das fêmeas (ZUCCHI, 2000).

Para fins de comparação das populações das espécies coletadas nas armadilhas, foram utilizados os seguintes índices faunísticos: frequência, dominância, constância, riqueza de espécies, e os índices de diversidade de Simpson, Shannon-Wiener e equitabilidade de Hill (modificado). Estes índices foram obtidos de acordo com Aguiar-Menezes et al. (2008). Estes índices não foram calculados para as moscas-das-frutas coletadas de frutos, pois as coletas foram ocasionais. 


\section{RESULTADOS E DISCUSSÃO}

Durante os dois anos de coleta, foi capturado, através de armadilhas, um total de 10.775 exemplares de moscas-das-frutas, sendo 8.875 C. capitata (2.639 machos e 6.236 fêmeas) e 1.900 de Anastrepha spp. (497 machos e 1.403 fêmeas), representadas por $A$. zenildae, A. sororcula, Anastrepha obliqua (Macquart) e Anastrepha dissimilis Stone (Tabela 1). Dos frutos de goiaba coletados aleatoriamente no pomar, emergiram C. capitata (44 exemplares), A. zenildae (28 exemplares) e $A$. sororcula (12 exemplares). Estas cinco espécies de moscas-das-frutas já foram relatadas para o Estado do Rio Grande do Norte. Segundo Araujo et al. (2005), mais de dez espécies de moscas-das-frutas ocorrem no Rio Grande do Norte. No entanto, estes são os primeiros registros de moscas-das-frutas na região central do Estado.

A riqueza e a diversidade de espécies observadas em Cruzeta $(\mathrm{RN})$ são semelhantes à encontrada em pomares de goiabeira localizados em outras regiões semiáridas do Brasil, como no norte de Minas Gerais, região sudeste (ALVARENGA et al., 2009) e Russas (CE), região nordeste (ARAUJO et al., 2008). Contudo, esta riqueza foi inferior à observada em um pomar de goiabeira no litoral sul da região nordeste, em Una (BA), onde 13 espécies de Anastrepha foram capturadas (DUTRA et al., 2009). Segundo estes autores, a diversidade de moscas-das-frutas encontrada em Una está relacionada, principalmente, com o fato de o referido pomar ser próximo a uma área de Mata Atlântica, onde provavelmente há muitos hospedeiros nativos e também a existência de outras frutíferas na proximidade do pomar. Dessa forma, a menor riqueza de espécies observada no presente trabalho pode estar relacionada com um fato mencionado por Araujo et al. (2005), que é a baixa diversidade de hospedeiros de moscas-das-frutas encontrada na Caatinga, vegetação típica do semiárido brasileiro. Além disso, havia um reduzido número de outras frutíferas próximo ao pomar.

Ocorreu um maior número de capturas e frequência de $C$. capitata, A. zenildae e $A$. sororcula, com destaque para a primeira, enquanto $A$. obliqua e $A$. dissimilis foram obtidas em menor número e frequência (Tabela 1). Ceratitis capitata, A. zenildae e A. sororcula também foram obtidas diretamente de frutos coletados no pomar, confirmando que estas espécies utilizam a goiaba como hospedeiro nesta região. Assim, constata-se, pela primeira vez, que $C$. capitata se encontra estabelecida na região do Seridó, Rio Grande do Norte. Araujo et al. (2005) relataram que, antes da década de 1990, nenhum exemplar da mosca-do-mediterrâneo havia sido detectado no
Rio Grande do Norte; contudo, menos de vinte anos após a primeira detecção, esta espécie agora infesta intensamente várias espécies de frutíferas no referido Estado. O menor número e a frequência de $A$. obliqua e $A$. dissimilis possivelmente estão relacionados com o fato de que goiaba não é hospedeiro primário para a primeira espécie e não é hospedeiro da segunda. Anastrepha dissimilis tem apenas plantas de Passifloraceae como hospedeiros conhecidos (ZUCCHI, 2007).

De maneira geral, constatou-se que C. capitata foi a única espécie dominante no pomar e também foi considerada constante, apesar de ter sido acessória em 2009. Anastrepha zenildae e A. sororcula foram consideradas constantes, $A$. obliqua foi acessória e $A$. dissimilis acidental (Tabela 1). $\mathrm{O}$ fato de $C$. capitata ter sido dominante e constante é interessante, pois difere de outros estudos realizados no semiárido, como, por exemplo, nos trabalhos desenvolvidos no norte de Minas Gerais (ALVARENGA et al., 2009) e Russas (CE) (ARAUJO et al., 2008), onde poucos exemplares de $C$. capitata foram capturados nos pomares de goiabeira. Nos pomares de goiabeira da região Nordeste do Brasil, geralmente $A$. zenildae é mais frequente e dominante, pois é bem adaptada às condições do semiárido (ARAUJO et al., 2005; ALVARENGA et al., 2009). Anastrepha zenildae é considerada uma praga importante de goiaba nos pomares localizados no semiárido (ARAÚJO et al., 2005). No entanto, vale lembrar que, em goiabeiras localizadas em centros urbanos, $C$. capitata pode ser uma espécie comum e predominante (ALVARENGA et al., 2010). Isto se deve ao fato de que, nos centros urbanos, há muitas frutíferas exóticas que são as preferidas por C. capitata (ARAUJO et al., 2005), permitindo a sucessão hospedeira e elevada população desta praga nos pomares.

Com relação aos pomares de goiabeira localizados em regiões geográficas mais distantes, com clima e flora diferentes do semiárido, os resultados diferem ainda mais. Raga et al. (2006) relataram que, em Monte Alegre do Sul (SP), região sudeste, A. fraterculus é a espécie de tefritídeo mais frequente em goiaba e que $C$. capitata não é comum. Dutra et al. (2009) relataram que $A$. fraterculus também foi mais frequente, dominante e constante em um pomar de goiabeira no município de Una (BA). Nos estados da região Norte, onde predomina a Floresta Amazônica, A. striata é a mais comum em goiaba (PEREIRA et al., 2010).

A elevada população de C. capitata no pomar de goiabeira, em Cruzeta (RN), semiárido brasileiro, possivelmente se deve a um conjunto de fatores, como, por exemplo, condições climáticas 
favoráveis à espécie e, principalmente, a forma de manejo do pomar, pois os nove hectares da área estudada eram divididos em parcelas menores e sempre havia plantas em diferentes fases de frutificação, permitindo a manutenção da população da mosca-do-mediterrâneo.

Neste estudo, o índice de Simpson foi considerado alto $(0,69)$ e indicou que a probabilidade de dois indivíduos escolhidos ao acaso e independentes pertencerem à mesma espécie foi de $69 \%$ (Tabela 1). Em relação ao índice de Shannon-Wiener, o baixo valor $(0,59)$ evidenciou a baixa diversidade da comunidade estudada, e quanto ao índice de equitatividade (Hill modificado), o baixo valor $(0,56)$ indicou que a distribuição das frequências entre as espécies coletadas não foi uniforme. Isso se deve à alta frequência e dominância de C. capitata.

Estudos realizados anteriormente, utilizando estes mesmos índices faunísticos, obtiveram alto valor para o índice de Simpson e baixos valores para os índices de Shannon-Wiener e equitatividade (Hill modificado). Um desses estudos foi realizado em Una (BA) (DUTRA et al., 2009), onde os valores obtidos foram semelhantes devido à alta frequência e dominância de $A$. fraterculus, espécie que representou $77 \%$ dos exemplares coletados. Os índices faunísticos obtidos no presente estudo também foram semelhantes aos dos estudos citados acima, contudo C. capitata foi frequente e dominante.

Os resultados obtidos no presente trabalho demonstram que $C$. capitata pode tornar-se praga-chave em pomares de goiabeira, nas condições do semiárido brasileiro. A maioria dos estudos publicados até o momento apresenta as espécies de Anastrepha como predominantes em pomares de goiabeira localizados no semiárido brasileiro. Outro aspecto relevante é que $C$. capitata, uma espécie exótica introduzida na década de 90 , na região Nordeste do Brasil, está estabelecendo-se em diferentes pontos do Nordeste, importante área de fruticultura do País. Essa constatação reafirma a necessidade de se conhecer as espécies de moscas-das-frutas antes de se implantar sistemas de manejo integrado destas pragas nos pomares, pois as espécies predominantes podem diferir até mesmo em pomares localizados em um mesmo ecossistema.

TABELA 1 - Caracterização de espécies de Anastrepha e Ceratitis em um pomar de goiabeira por índices faunísticos, no município de Cruzeta (RN), de janeiro/2008 a dezembro/2009.

\begin{tabular}{|c|c|c|c|c|c|c|}
\hline \multirow{2}{*}{ Espécies } & \multicolumn{3}{|c|}{$\mathrm{N}^{1}$} & \multicolumn{3}{|c|}{ Frequência (\%) } \\
\hline & Total & 2008 & 2009 & Total & 2008 & 2009 \\
\hline C. capitata & 6.236 & 1.510 & 4.726 & 81,6 & 65,6 & 88,6 \\
\hline A. zenildae & 1.156 & 643 & 513 & 15,1 & 27,9 & 9,6 \\
\hline A. sororcula & 179 & 100 & 79 & 2,3 & 4,3 & 1,5 \\
\hline A. obliqua & 51 & 43 & 8 & 0,7 & 1,9 & 0,2 \\
\hline A. dissimilis & 17 & 6 & 11 & 0,2 & 0,3 & 0,2 \\
\hline Riqueza (S) & 5 & 5 & 5 & & & \\
\hline Índice de Simpson & 0,69 & 0,51 & 0,79 & & & \\
\hline Índice de Shannon & 0,59 & 0,93 & 0,44 & & & \\
\hline Índice de Hill modificado & 0,56 & 0,62 & 0,48 & & & \\
\hline \multirow{2}{*}{ Espécies } & \multicolumn{3}{|c|}{ Dominância $^{2}$} & \multicolumn{3}{|c|}{ Constância $^{3}$} \\
\hline & Total & 2008 & 2009 & Total & 2008 & 2009 \\
\hline C. capitata & $\mathrm{d}$ & $\mathrm{d}$ & $\mathrm{d}$ & $71,6(w)$ & $90,2(w)$ & $50,0(y)$ \\
\hline A. zenildae & $\mathrm{n}$ & $\mathrm{d}$ & $\mathrm{n}$ & 73,7 (w) & $60,8(w)$ & $88,6(w)$ \\
\hline A. sororcula & $\mathrm{n}$ & $\mathrm{n}$ & $\mathrm{n}$ & $51,6(w)$ & $58,8(w)$ & $43,2(\mathrm{y})$ \\
\hline A. obliqua & $\mathrm{n}$ & $\mathrm{n}$ & $\mathrm{n}$ & $29,5(\mathrm{y})$ & 41,2 (y) & $15,9(\mathrm{z})$ \\
\hline A. dissimilis & $\mathrm{n}$ & $\mathrm{n}$ & $\mathrm{n}$ & $9,5(\mathrm{z})$ & $7,84(\mathrm{y})$ & $11,4(\mathrm{z})$ \\
\hline
\end{tabular}

$\mathrm{N}^{1}=$ Total de fêmeas capturadas; ${ }^{2} \mathrm{~d}$ : dominante; n: não dominante; ${ }^{3} \mathrm{w}$ : constante; y: acessórias; $\mathrm{z}$ : acidental; Total: 2008-2009. 


\section{CONCLUSÕES}

Ceratitis capitata, Anastrepha zenildae, Anastrepha sororcula, Anastrepha obliqua e Anastrepha dissimilis são as espécies constatadas no pomar de goiabeira estudado, em Cruzeta (RN). Dessas, C. capitata, A. zenildae e A. sororcula são obtidas diretamente dos frutos de goiaba. Verifica-se que C. capitata é a espécie mais frequente, constante e dominante, no referido pomar. As informações chamam a atenção para o fato de $C$. capitata ser uma espécie comum em goiaba, nas condições do semiárido brasileiro.

\section{AGRADECIMENTOS}

Ao técnico Ernesto Espínola Sobrinho (EMPARN), pela coleta do material em campo. A Isadora M. Paiva de Lima e Wigna G. N. Santos (estudantes do curso de Agronomia da UFERSA), pelo auxílio na catalogação dos resultados. Ao BNB (Banco do Nordeste do Brasil), pelo apoio financeiro.

\section{REFERÊNCIAS}

AGUIAR-MENEZES, E.L.; SOUZA, S.A.S.; LIMAFILHO, M.; BARROS, H.C.; FERRARA, F.A.A.; MENEZES, E.B. Análise faunística de moscas-dasfrutas (Diptera: Tephritidae) nas regiões Norte e Noroeste do Estado do Rio de Janeiro. Neotropical Entomology, Londrina, v.37, n.1 p.8-14, 2008.

ALVARENGA, C.D.; ALVES, D.A.; SILVA, M.A.; LOPES, E.N.; LOPES, G.N. Moscas-das-frutas (Diptera: Tephritidae) em pomares da área urbana no norte de Minas Gerais. Revista Caatinga, Mossoró, v.23, n.2, p.25-31, 2010.

ALVARENGA, C.D.; MATRANGOLO, C.A.R.; LOPES, G.N.; SILVA, M.A.; LOPES, E.N.; ALVES, D.A.; NASCIMENTO, A.S.; ZUCCHI, R.A. Moscasdas-frutas (Diptera: Tephritidae) e seus parasitóides em plantas hospedeiras de três municípios do norte do estado de Minas Gerais. Arquivos do Instituto Biológico, São Paulo, v.76, n.2, p.195-204, 2009.

AMORIM, I.L.; SAMPAIO, E.V.S.B.; ARAÚJO, E.L. Flora e estrutura da vegetação arbustiva-arbórea de uma área de caatinga do Seridó, RN, Brasil. Acta Botânica Brasílica, São Paulo, v.19, n.3, p.615-623, 2005.
ARAUJO, E.L.; MEDEIROS, M.K.M.; SILVA, V.E.; ZUCCHI, R.A. Moscas-das-frutas (Diptera: Tephritidae) no semi-árido do Rio Grande do Norte: plantas hospedeiras e índices de infestação. Neotropical Entomology, Londrina, v.34, n.6, p.889-894, 2005.

ARAUJO, E.L.; SILVA, R.K.; GUIMARÃES, J.A.; SILVA, J.G.; BITTENCOURT, M.A.L. Levantamento e flutuação populacional de moscasdas-frutas (Diptera: Tephritidae) em goiaba Psidium guajava L., no município de Russas (CE). Revista Caatinga, Mossoró, v.21, n.1, p.138-146, 2008.

BIRK, A.; ALUJA, M. Anastrepha ludens and Anastrepha serpentina (Diptera: Tephritidae) do not infest Psidium guajava (Myrtaceae), but Anastrepha obliqua occasionally shares this resource with Anastrepha striata in nature. Journal of Economic Entomology, Lanham, v.104, n.4, p.1204-1211, 2011.

DUTRA, V.S.; SANTOS, M.S.; SOUZA FILHO, Z.A.; ARAUJO, E.L.; SILVA, J.G. Faunistic analysis of Anastrepha spp. (Diptera: Tephritidae) on a guava orchard under organic management in the municipality of Una, Bahia, Brasil. Neotropical Entomology, Londrina, v.38, n.1, p.133-138, 2009.

NICÁCIO, J.; UCHÔA, M.A. Diversity of frugivorous flies (Diptera: Tephritidae and Lonchaeidae) and their relationship with host plants (angiospermae) in environments of south pantanal region, Brasil. Florida Entomologist, Gainesville, v.94, n.3, p.443466, 2011.

PEREIRA, F. M.; RYOSUKE, K. Contribuição da pesquisa científica brasileira no desenvolvimento de algumas frutíferas de clima subtropical. Revista Brasileira de Fruticultura. Jaboticabal, v. 33. n. 1- Edição Especial. p. 92-108, 2011.

PEREIRA, J.D.B.; BURITI, D.P.; LEMOS, W.P., SILVA, W.R.; SILVA, R.A. Espécies de Anastrepha Schiner (Diptera: Tephritidae), seus hospedeiros e parasitóides nos estados do Acre e Rondônia, Brasil. Biota Neotropical, São Paulo, v.10, n.3, p.441-446, 2010

RAGA, A.; SOUZA FILHO, M.F. de; PRESTES, D.A.O.; AZEVEDO FILHO, J.A. de; SATO, 
M.E. Susceptibility of guava genotypes to natural infestation by Anastrepha spp. (Diptera: Tephritidae) in the municipality of Monte Alegre do Sul, state of São Paulo, Brazil. Neotropical Entomology, Londrina, v.35, n.1, p.121-125, 2006.

SILVA, J.G.; DUTRA, V.S.; SANTOS, M.S.; SILVA, N.M.O.; VIDAL, D.B.; NINK, R.A.; GUIMARAES, J.A.; ARAUJO, E.L. Diversity of Anastrepha spp. (Diptera: Tephritidae) and associated braconid parasitoids from native and exotic hosts in Southeastern Bahia, Brazil. Environmental Entomology, Lanham, v.39, n.5, p.1457-1465, 2010.

SILVA, L.N.; SANTOS, M.S.; DUTRA, V.S.; ARAUJO, E.L.; COSTA, M.A.; SILVA, J.G. First survey of fruit fly (Diptera: Tephritidae) and parasitoid diversity among Myrtaceae fruit across the state of Bahia, Brazil. Revista Brasileira de Fruticultura, Jaboticabal, v.33, n.3, p.757-764, 2011.

SINGH, S.P.; PAL, R.K. Controlled atmosphere storage of guava (Psidium guajava L.) fruit. Postharvest Biology and Technology, Langford Lane, v.47, p.296-306, 2008.
SOUZA FILHO, M.F.; RAGA, A.; AZEVEDO FILHO, J.A.; STRIKIS, P.C.; GUIMARÃES, J.A.; ZUCCHI, R.A. Diversity and seasonality of fruit flies (Diptera: Tephritidae and Lonchaeidae) and their parasitoids (Hymenoptera: Braconidae and Figitidae) in orchards of guava, loquat and peach. Brazilian Journal of Biology, São Carlos, v.69, p.31-40, 2009.

ZUCCHI, R.A. Taxonomia. In: MALAVASI, A.; ZUCCHI, R.A. (Ed.). Moscas-das-frutas de importância econômica no Brasil: conhecimento básico e aplicado. Ribeirão Preto: Holos Editora, 2000. p.13-24.

ZUCCHI, R.A. Diversidad, distribución y hospederos del género Anastrepha en Brasil. In HERNÁNDEZ-ORTIZ, V. (Ed.). Moscas de la fruta en Latinoamérica (Diptera: Tephritidae): diversidad, biología y manejo. Mexico: S y G Editores, 2007. p.77-100. 